\title{
Highly Diastereoselective Catalytic MPV Reductions
}

\author{
Jingjun Yin, ${ }^{*}$ Mark A. Huffman, Karen M. Conrad, Joseph, D. Armstrong, III
}

Department of Process Research, Merck Research Laboratories, P. O. Box 2000, Rahway, NJ 07065

jingjun_yin@merck.com

\section{Supporting Information}

Table of Contents

1. General comments............................................... 2

2. General Procedure for MPV reduction in Tables 2 and $3 \ldots \ldots \ldots \ldots \ldots \ldots . . . . . . \ldots 2$

3. General Procedure for $\mathrm{NaBH}_{4}$ reduction in Tables 2 and $3 \ldots \ldots \ldots \ldots \ldots \ldots . . . . . . . .33$

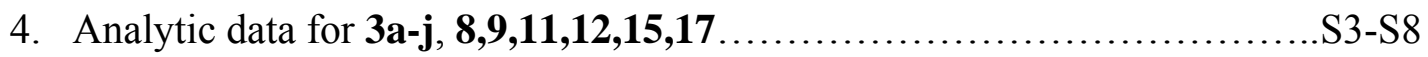

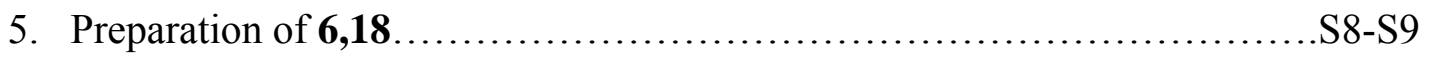

6. Analytic data for $\mathbf{3 a - j , ~} \mathbf{8 , 9 , 1 1 , 1 2 , 1 5 , 1 7 \ldots \ldots \ldots \ldots \ldots \ldots \ldots \ldots \ldots \ldots \ldots \ldots . . S 9 - S 1 0}$

7. References...........................................................

8. Copies of NMR spectra of 3d,3f................................. 1112 
General Comments: An HPLC fitted with an Ace C-8 column, auto-injector and diode array detector provided information on reaction progress and assay yield. Relevant HPLC parameters include $0.1 \% \mathrm{H}_{3} \mathrm{PO}_{4}$ in $\mathrm{H}_{2} \mathrm{O} / \mathrm{MeCN}$ mobile phase, $35{ }^{\circ} \mathrm{C}$ column temperature, and monitoring at $210 \mathrm{~nm}$. A ChiralPak AD-H (4.6 x $150 \mathrm{~mm})$ column was used for measuring the ee's of compound 3a,e with $0.1 / 10 / 90$ of $\mathrm{TFA} / \mathrm{iPrOH} / \mathrm{heptane}$ as the mobile phase and a flow rate of $1.5 \mathrm{~mL} / \mathrm{min}$ at $30{ }^{\circ} \mathrm{C}$. A $400 \mathrm{MHz}$ NMR system captured all ${ }^{1} \mathrm{H},{ }^{13} \mathrm{C}$ and ${ }^{19} \mathrm{~F}$ spectral data associated with all compounds. All columns were packed with $40-63 \mu \mathrm{m}$ silica gel. Ketones $2 \mathbf{a}-\mathbf{h}$ were prepared according to the slightly modified literature procedure from aryl Gringards. ${ }^{1}$ Other ketones are commercially available.

General Procedure for MPV reduction in Tables 2 and 3: A mixture of ketone ( 0.637 mmol, 1.0 equiv), $\mathrm{Al}(\mathrm{OiPr})_{3}(0.2$ to 0.6 equiv), and $i \mathrm{PrOH}(0.536 \mathrm{~mL}, 11$ equiv) in toluene $(0.80 \mathrm{~mL}, 1.3 \mathrm{~mL} / \mathrm{mmol})$ was heated at $50{ }^{\circ} \mathrm{C}$ under $\mathrm{N}_{2}$ until $\mathrm{LC}$ revealed complete conversion. Unless specified otherwise, the reaction was quenched with $4 \mathrm{~mL}$ of $1 \mathrm{~N} \mathrm{HCl}$ and $4 \mathrm{~mL}$ of EtOAc. The organic layer was washed with $4 \mathrm{~mL}$ of water and concentrated. Crude ${ }^{1} \mathrm{H}$ NMR was taken to determine the diastereoselectivity. The product was further purified to $>95 \%$ pure by $\mathrm{LC}$ with a d.r. of $>50 / 1$ by $\mathrm{SiO}_{2}$ column chromatography or a hexane tituration if necessary.

General Procedure for $\mathrm{NaBH}_{4}$ reduction in Tables 2 and 3: A solution of ketone (60mg) in $\mathrm{MeOH}(0.5 \mathrm{~mL})(0.5 \mathrm{~mL}$ THF was added to help dissolve the ketone in some cases) at rt was treated with 2 equiv of solid $\mathrm{NaBH}_{4}$. After 5-30 min when LC reveals complete conversion, the reaction was quenched with $4 \mathrm{~mL} 1 \mathrm{~N} \mathrm{HCl}$ and $4 \mathrm{~mL}$ EtOAc. 
The organic layer was washed with $4 \mathrm{~mL}$ water and concentrated. NMR was taken of the crude material, which was always pure with only two product isomers.

\section{(1R,2S)-1-(3',5'-Bistrifluoromethylphenyl)-2-benzyloxycarbonylamino-1-propanol}

(3a) The general procedure provided 3a, $260 \mathrm{mg}$ at $97 \%$ isolated yield as a white solid with $>99 \%$ ee (retention times: desired 1R,2S-enantiomer, $2.6 \mathrm{~min}$; undesired, $4.7 \mathrm{~min}$ ). M.p. $141-142{ }^{\circ} \mathrm{C} ; \quad{ }^{1} \mathrm{H}$ NMR $\left(400 \mathrm{MHz}, \mathrm{CDCl}_{3}\right) . \delta 7.82(\mathrm{~d}, J=9.6 \mathrm{~Hz}, 3 \mathrm{H}), 7.33-7.40$ (m, 5 H), 5.14 (s, 2 H), 5.05 (br s, 1 H), 4.95 (br d, J = 4.4 Hz, 1 H), 4.06 (br s, 1 H), 3.40 (br s, $1 \mathrm{H}), 1.01(\mathrm{~d}, J=6.9 \mathrm{~Hz}, 3 \mathrm{H}) ;{ }^{13} \mathrm{C} \mathrm{NMR}\left(100 \mathrm{MHz}, \mathrm{CDCl}_{3}\right) \delta 156.7,143.5,136.0$, $131.5(\mathrm{q}, J=33.3 \mathrm{~Hz}), 128.6,128.4,128.1,126.5(\mathrm{~m}), 123.3(\mathrm{q}, J=273 \mathrm{~Hz}), 121.5(\mathrm{~m})$, 75.3, 67.3, 52.5, 13.9. Anal. Calcd. for $\mathrm{C}_{19} \mathrm{H}_{17} \mathrm{~F}_{6} \mathrm{NO}_{3}$ : C, 54.16; H, 4.07; N, 3.32. Found: C, 54.15; H, 3.84; N, 3.29.

Characteristic ${ }^{1} \mathrm{H}$ NMR signals for the minor isomer: $1.21(\mathrm{~d}, J=6.9 \mathrm{~Hz}, 3 \mathrm{H})$.

(1R,2S)-1-Phenyl-2-benzyloxycarbonylamino-1-propanol (3b) ${ }^{2}$ The general procedure provided 3b, $174 \mathrm{mg}$ at $95 \%$ isolated yield as a white solid. M.p. $115-116^{\circ} \mathrm{C}$ (lit. m.p. ${ }^{2}$ 111-113 $\left.{ }^{\circ} \mathrm{C}\right) ; \quad{ }^{1} \mathrm{H}$ NMR (400 MHz, $\left.\mathrm{CDCl}_{3}\right) . \delta 7.15-7.55(\mathrm{~m}, 10 \mathrm{H}), 5.12(\mathrm{~s}, 2 \mathrm{H}), 5.08$ (br d, $J=8.0 \mathrm{~Hz}, 1 \mathrm{H}), 4.88$ (br s, $1 \mathrm{H}), 4.06$ (br s, $1 \mathrm{H}), 3.0$ (br s, $1 \mathrm{H}), 1.00$ (d, $J=-6.9$ $\mathrm{Hz}, 3 \mathrm{H}) ;{ }^{13} \mathrm{C} \mathrm{NMR}\left(100 \mathrm{MHz}, \mathrm{CDCl}_{3}\right) \delta 156.5,140.7,136.4,128.5,128.24,128.17$, 128.12, 127.5, 126.2, 76.2, 66.9, 52.4, 14.4; Anal. Calcd. for $\mathrm{C}_{17} \mathrm{H}_{19} \mathrm{NO}_{3}: \mathrm{C}, 71.56$; $\mathrm{H}$, $6.71 ; \mathrm{N}, 4.91$ Found: C, 71.26; H, 6.70; N, 4.82 .

Characteristic ${ }^{1} \mathrm{H}$ NMR signals for the minor isomer: $1.12(\mathrm{~d}, J=6.8 \mathrm{~Hz}, 3 \mathrm{H})$. (1R,2S)-1-p-Tolyl-2-benzyloxycarbonylamino-1-propanol (3c) The general procedure provided 3c, $172 \mathrm{mg}$ at $90 \%$ isolated yield as a white solid. M.p. $120-121{ }^{\circ} \mathrm{C} ;{ }^{1} \mathrm{H}$ NMR $\left(400 \mathrm{MHz}, \mathrm{CDCl}_{3}\right) . \delta$ 7.32-7.38 (m, $\left.5 \mathrm{H}\right), 7.14-7.24(\mathrm{~m}, 4 \mathrm{H}), 5.12(\mathrm{~s}, 2 \mathrm{H}), 5.03$ (br d, J 
$=7.6 \mathrm{~Hz}, 1 \mathrm{H}), 4.85(\mathrm{br} \mathrm{s}, 1 \mathrm{H}), 4.04(\mathrm{br} \mathrm{s}, 1 \mathrm{H}), 2.67(\mathrm{br} \mathrm{s}, 1 \mathrm{H}), 2.35(\mathrm{~s}, 3 \mathrm{H}), 1.01(\mathrm{~d}, J$ $=6.9 \mathrm{~Hz}, 3 \mathrm{H}) ;{ }^{13} \mathrm{C} \mathrm{NMR}\left(100 \mathrm{MHz}, \mathrm{CDCl}_{3}\right) \delta 156.5,137.8,137.3,136.6,129.0,128.6$, 128.21, 128.17, 126.2, 76.3, 66.9, 52.5, 21.2, 14.6; Anal. Calcd. for $\mathrm{C}_{18} \mathrm{H}_{21} \mathrm{NO}_{3}$ : C, 72.22 ; H, 7.07; N, 4.68; Found: C, 71.83; H, 7.04; N, 4.57.

Characteristic ${ }^{1} \mathrm{H}$ NMR signals for the minor isomer: 2.37 (s, $\left.3 \mathrm{H}\right), 1.12(\mathrm{~d}, J=6.8 \mathrm{~Hz}, 3$ H).

(1R,2S)-1-o-Tolyl-2-benzyloxycarbonylamino-1-propanol (3d) The general procedure provided 3d, $164 \mathrm{mg}$ at $86 \%$ isolated yield as a colorless oil. ${ }^{1} \mathrm{H}$ NMR (400 MHz, $\mathrm{CDCl}_{3}$ ) $\delta$ 7.51-7.53 (m, $1 \mathrm{H}), 7.36-7.37$ (m, 5 H), 7.16-7.24 (m, $3 \mathrm{H}), 5.44(\mathrm{br} \mathrm{d}, J=7.6 \mathrm{~Hz}, 1 \mathrm{H})$, 5.08-5.16 (m, 3 H), 3.99 (br s, 1 H), 2.98 (br s, 1 H), 2.40 (br s, 2.4 H) + 2.14 (br s, 0.6 H), $1.02(\mathrm{~d}, J=6.7 \mathrm{~Hz}, 3 \mathrm{H}) ;{ }^{13} \mathrm{C} \mathrm{NMR}\left(100 \mathrm{MHz}, \mathrm{CDCl}_{3}\right) \delta 156.1,139.3,136.5,124.7$, $130.4,128.6,128.4,128.16,128.14,127.3,126.1,125.8,72.6,66.7,50.5,19.0,13.2$. HRMS $\mathrm{C}_{18} \mathrm{H}_{21} \mathrm{NO}_{3}$ calcd for [M+Na]: 322.14136, found 322.14234.

Characteristic ${ }^{1} \mathrm{H}$ NMR signals for the minor isomer: $1.20(\mathrm{~d}, J=6.8 \mathrm{~Hz}, 3 \mathrm{H})$.

(1R,2S)-1-(2'-Methoxyphenyl)-2-benzyloxycarbonylamino-1-propanol (3e) The general procedure provided 3e, $193 \mathrm{mg}$ at $96 \%$ isolated yield as a colorless oil with $>99 \%$ ee (retention times: desired 1R,2S-enantiomer, $9.9 \mathrm{~min}$; undesired, $11.3 \mathrm{~min}$ ). ${ }^{1} \mathrm{H}$ NMR $\left(400 \mathrm{MHz}, \mathrm{CDCl}_{3}\right) . \delta 7.24-7.39(\mathrm{~m}, 7 \mathrm{H}), 6.96(\mathrm{t}, J=7.4 \mathrm{~Hz}, 1 \mathrm{H}), 6.86(\mathrm{~d}, J=8.0 \mathrm{~Hz}, 1$ H), 5.21 (br m, 1 H), 5.08 (br s, 3 H), $4.13($ br s, 1 H), 3.83 (br s, 2.4 H) + 3.67 (br s, 0.6 H), $2.96($ br s, $1 \mathrm{H}), 1.05(\mathrm{~d}, J=6.8 \mathrm{~Hz}, 3 \mathrm{H}) ;{ }^{13} \mathrm{C}$ NMR $\left(100 \mathrm{MHz}, \mathrm{CDCl}_{3}\right) \delta 156.5$, $156.2,136.7,129.1,128.60,128.55,128.1,127.7,120.7,110.5,72.8,66.6,55.4,51.2$, 15.2; Anal. Calcd. for $\mathrm{C}_{18} \mathrm{H}_{21} \mathrm{NO}_{4}$ : C, 68.55; H, 6.71; N, 4.44; Found: C, 68.28; H, 6.74; N, 4.36 . 
Characteristic ${ }^{1} \mathrm{H}$ NMR signals for the minor isomer: $1.19(\mathrm{~d}, J=6.7 \mathrm{~Hz}, 3 \mathrm{H})$.

(1R,2S)-1-(5'-Methoxy-3'-pyridyl)-2-benzyloxycarbonylamino-1-propanol (3f) The general procedure provided a reaction mixture of $3 f$. It was filtered with $1 \mathrm{~mL}$ toluene rinse to give the first crop of product $(106 \mathrm{mg})$. The filtrate was passed through a pad of silica gel $(2 \times 2 \mathrm{~cm})$ with EtOAc $(130 \mathrm{~mL})$, concentrated, and crystallized from $3 \mathrm{~mL}$ toluene to give the second crop of product (58 mg, combined $164 \mathrm{mg}, 82 \%$ yield) as an off-white solid. M.p. $141-143{ }^{\circ} \mathrm{C} ;{ }^{1} \mathrm{H}$ NMR (400 MHz, $\left.\mathrm{CDCl}_{3}\right) . \delta 8.18$ (br s, $2 \mathrm{H}$ ), 7.19$7.39(\mathrm{~m}, 6 \mathrm{H}),(5.12-5.15(\mathrm{~m}, 3 \mathrm{H}), 4.93$ (br s, $1 \mathrm{H}), 4.06$ (br s, $1 \mathrm{H}), 3.83(\mathrm{~s}, 3 \mathrm{H}), 1.04$ $(\mathrm{d}, J=6.8 \mathrm{~Hz}, 3 \mathrm{H}) ;{ }^{13} \mathrm{C} \mathrm{NMR}\left(100 \mathrm{MHz}, \mathrm{CDCl}_{3}\right) \delta 156.7,155.8,140.0,137.5,136.6$, 136.3, 128.7, 128.3, 128.2, 118.7, 74.2, 67.1, 55.6, 52.5, 14.5. HRMS $\mathrm{C}_{17} \mathrm{H}_{20} \mathrm{~N}_{2} \mathrm{O}_{4}$ calcd for $[\mathrm{M}+\mathrm{Na}]:$ 339.13153, found 339.13251.

Characteristic ${ }^{1} \mathrm{H}$ NMR signals for the minor isomer: $1.15(\mathrm{~d}, J=6.8 \mathrm{~Hz}, 3 \mathrm{H})$.

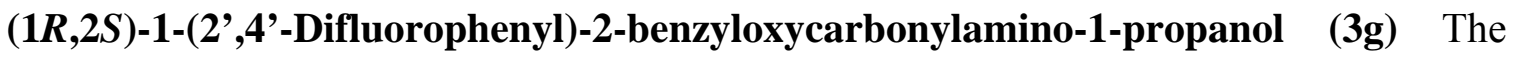
general procedure provided 3g, $190 \mathrm{mg}$ at 93\% isolated yield as a white solid. M.p. 68$69{ }^{\circ} \mathrm{C} ;{ }^{1} \mathrm{H}$ NMR $\left(400 \mathrm{MHz}, \mathrm{CDCl}_{3}\right) . \delta$ 7.32-7.37 (m, $\left.4 \mathrm{H}\right)$, 7.19-7.24 (m, $\left.2 \mathrm{H}\right), 6.91-6.96$ (m, 2 H), 5.09-5.13 (br m, 4 H), 4.10 (br s, 1 H), 3.48 (br s, $1 \mathrm{H}), 1.02$ (d, J = 6.8 Hz, 3 $\mathrm{H}) ;{ }^{13} \mathrm{C} \mathrm{NMR}\left(100 \mathrm{MHz}, \mathrm{CDCl}_{3}\right) \delta 158.5(\mathrm{dd}, J=242,1.7 \mathrm{~Hz}), 156.7,155.4(\mathrm{~d}, J=245$ $\mathrm{Hz}), 136.2,130.0$ (m, 1 C), 128.5, 128.2, 128.1, 116.2 (dd, $J=25,8.6 \mathrm{~Hz}), 115.3$ (dd, $J=$ 24, 8.6 Hz), $114.8(\mathrm{dd}, J=25,1.7 \mathrm{~Hz}), 70.3,67.0,51.3,14.6 ;{ }^{19} \mathrm{~F}$ NMR $(377 \mathrm{MHz}$, $\left.\mathrm{CDCl}_{3}\right) \delta$-118.6, -123.7. Anal. Calcd. for $\mathrm{C}_{17} \mathrm{H}_{17} \mathrm{~F}_{2} \mathrm{NO}_{3}$ : C, 63.55; H, 5.33; N, 4.36. Found: C, 63.51; H, 5.30; N, 4.31.

Characteristic ${ }^{1} \mathrm{H}$ NMR signals for the minor isomer: $1.23(\mathrm{~d}, J=6.8 \mathrm{~Hz}, 3 \mathrm{H})$. 
(1R,2S)-1-(4'-Cyanophenyl)-2-benzyloxycarbonylamino-1-propanol (3h) The general procedure provided $3 \mathbf{h}, 180 \mathrm{mg}$ at $91 \%$ isolated yield as a white solid. M.p. $93-95{ }^{\circ} \mathrm{C} ;{ }^{1} \mathrm{H}$ NMR (400 MHz, $\left.\mathrm{CDCl}_{3}\right) \delta 7.58(\mathrm{~d}, J=8.0 \mathrm{~Hz}, 2 \mathrm{H}), 7.45(\mathrm{~d}, J=8.0 \mathrm{~Hz}, 2 \mathrm{H}), 7.34-7.37$ (m, 5 H), 5.11 (s, 2 H), 4.93 (s, 1 H), 4.02 (br s, 1 H), 3.52 (br s, 1 H), 0.98 (d, J = 6.8 Hz, $3 \mathrm{H}) ;{ }^{13} \mathrm{C}$ NMR $\left(100 \mathrm{MHz}, \mathrm{CDCl}_{3}\right) \delta 156.5,146.4,136.1,132.0,128.6,128.3,128.1$, 127.0, 118.7, 111.2, 75.6, 67.1, 52.4, 14.1; Anal. Calcd. for $\mathrm{C}_{18} \mathrm{H}_{18} \mathrm{~N}_{2} \mathrm{O}_{3}$ : C, 69.66; $\mathrm{H}$, 5.85; N, 9.03. Found: C, 69.43; H, 5.82; N, 8.78.

Characteristic ${ }^{1} \mathrm{H}$ NMR signals for the minor isomer: $1.18(\mathrm{~d}, J=6.8 \mathrm{~Hz}, 3 \mathrm{H})$.

(2S,3S)-1-Chloro-3-benzyloxycarbonylamino-3-benzyl-2-propanol (3i) ${ }^{3}$ The general procedure provided 3i, $201 \mathrm{mg}$ at $94 \%$ isolated yield as a white solid. M.p. $152-153{ }^{\circ} \mathrm{C}$; ${ }^{1} \mathrm{H}$ NMR (400 MHz, CDCl $) . \delta$ 7.19-7.36 (m, $\left.10 \mathrm{H}\right), 5.05(\mathrm{~s}, 2 \mathrm{H}), 4.86(\mathrm{~d}, J=7.2 \mathrm{~Hz}, 1$ H), 3.97-4.04 (m, $1 \mathrm{H}), 3.87$ (br s, $1 \mathrm{H}), 3.67$ (dd, $J=11.2,3.8 \mathrm{~Hz}, 1 \mathrm{H}, 3.59$ (dd, $J=11.2$, 7.6 Hz, $1 \mathrm{H}), 2.91-3.03(\mathrm{~m}, 3 \mathrm{H}) ;{ }^{13} \mathrm{C}$ NMR $\left(100 \mathrm{MHz}, \mathrm{CDCl}_{3}\right) \delta 156.3,137.1,136.3$, $129.5,128.7,128.6,128.3,128.0,126.8,73.3,67.0,54.8,47.7,35.8$.

Characteristic ${ }^{1} \mathrm{H}$ NMR signals for the minor isomer: $5.18(\mathrm{~d}, J=8.8 \mathrm{~Hz}, 1 \mathrm{H}), 5.09(\mathrm{~s}, 2$ $\mathrm{H})$.

(2S,3S)-1-Chloro-3-p-tosylamino-3-benzyl-2-propanol (3j) The general procedure provided $225 \mathrm{mg}$ of an oil at $100 \%$ isolated yield for the combined isomers. Flash silica gel column gave $187 \mathrm{mg}$ of the main isomer $\mathbf{3 j}$ ( $83 \%$ yield) as a solid and $38 \mathrm{mg}$ of the minor isomer that was contaminated with some main isomer. Main isomer: M.p. 77-78 ${ }^{\circ} \mathrm{C} ;{ }^{1} \mathrm{H}$ NMR (400 MHz, $\left.\mathrm{CDCl}_{3}\right) \delta 8.53(\mathrm{~d}, J=8.2 \mathrm{~Hz}, 2 \mathrm{H}), 7.16-7.20(\mathrm{~m}, 5 \mathrm{H}), 6.96(\mathrm{~d}$, $J=7.2 \mathrm{~Hz}, 2 \mathrm{H}), 4.76(\mathrm{~d}, J=8.4 \mathrm{~Hz}, 1 \mathrm{H}), 3.85-3.90(\mathrm{~m}, 1 \mathrm{H}), 3.71(\mathrm{dd}, J=11.4,4.6 \mathrm{~Hz}$, $1 \mathrm{H}), 3.58-3.66(\mathrm{~m}, 2 \mathrm{H}), 2.82(\mathrm{dd}, J=14.0,7.6 \mathrm{~Hz}, 1 \mathrm{H}), 2.69(\mathrm{dd}, J=14.0,5.2 \mathrm{~Hz}, 1$ 
$\mathrm{H}), 2.42(\mathrm{~s}, 3 \mathrm{H}) ;{ }^{13} \mathrm{C}$ NMR $\left(100 \mathrm{MHz}, \mathrm{CDCl}_{3}\right) \delta 143.4,136.6,136.0,129.7,129.5$, 128.6, 126.9, 126.7, 72.6, 56.9, 46.6, 35.1, 21.5; Anal. Calcd. for $\mathrm{C}_{17} \mathrm{H}_{20} \mathrm{ClNO}_{3} \mathrm{~S}: \mathrm{C}$, 57.70; H, 5.70; N, 3.96. Found: C, 57.73; H, 5.70; N, 3.91.

Minor isomer (2R,3S)-1-Chloro-3-p-tosylamino-3-benzyl-2-propanol: ${ }^{1} \mathrm{H}$ NMR (400 $\left.\mathrm{MHz}, \mathrm{CDCl}_{3}\right) . \delta 7.70(\mathrm{~d}, J=8.0 \mathrm{~Hz}, 2 \mathrm{H}), 7.17-7.25(\mathrm{~m}, 5 \mathrm{H}), 7.01-7.04(\mathrm{~m}, 2 \mathrm{H}), 5.19$ $(\mathrm{d}, J=9.2 \mathrm{~Hz}, 1 \mathrm{H}), 3.67-3.71(\mathrm{~m}, 1 \mathrm{H}), 3.56-3.63(\mathrm{~m}, 2 \mathrm{H}), 3.47(\mathrm{dd}, J=11.2,4.0 \mathrm{~Hz}, 1$ H), $2.87(\mathrm{dd}, J=13.1,9.3 \mathrm{~Hz}, 1 \mathrm{H}), 2.62(\mathrm{dd}, J=13.1,5.6 \mathrm{~Hz}, 1 \mathrm{H}), 2.43(\mathrm{~s}, 3 \mathrm{H}) .{ }^{13} \mathrm{C}$ NMR $\left(100 \mathrm{MHz}, \mathrm{CDCl}_{3}\right) \delta 143.5,137.7,126.8,129.8,129.2,128.7,126.9,126.8,70.7$, $56.7,47.6,38.8,21.5$.

syn-2-Methoxy-1,2-diphenylethanol (8) ${ }^{4}$ The general procedure carried out in $5.0 \mathrm{mmol}$ scale provided syn-product 8 and its anti-isomer 9 in a ratio of 93.7/6.3, $1.15 \mathrm{~g}$ at $100 \%$ isolated yield as a white solid. M.p. $52-53.5^{\circ} \mathrm{C}$ (lit. ${ }^{5}$ syn- mp $53{ }^{\circ} \mathrm{C}$; anti- mp $100{ }^{\circ} \mathrm{C}$ ); $\mathrm{NaBH}_{4}$ reduction gave a syn/anti ratio of 8.3/91.7. ${ }^{1} \mathrm{H}$ NMR data for both isomers are in accord with the literature. ${ }^{4}$ GC-MS, $m / e 228\left(\mathrm{M}^{+}\right), 210\left(\mathrm{M}^{+}-\mathrm{H}_{2} \mathrm{O}\right)$.

syn-2-Ethoxy-1,2-diphenylethanol (11) ${ }^{6}$ The general procedure carried out in 2.55 mmol scale provided a mixture of syn-product $\mathbf{1 1}$ and its anti-isomer in a ratio of 95.5/4.5, $584 \mathrm{mg}$ at $95 \%$ isolated yield as an oil. $\mathrm{NaBH}_{4}$ reduction gave a syn/anti ratio of 12/88. ${ }^{1} \mathrm{H}$ NMR data for both isomers are in accord with the literature. ${ }^{6}$ GC-MS, $m / e$ $242\left(\mathrm{M}^{+}\right), 224\left(\mathrm{M}^{+}-\mathrm{H}_{2} \mathrm{O}\right)$.

syn-2-Isopropoxy-1,2-diphenylethanol (12) ${ }^{4}$ The general procedure carried out in 2.55 mmol scale provided syn-product 12 and its anti-isomer in a ratio of 96.1/3.9, 640 $\mathrm{mg}$ at $98 \%$ isolated yield as a colorless oil. $\mathrm{NaBH}_{4}$ reduction gave a syn/anti ratio of 21/79. ${ }^{1} \mathrm{H}$ 
NMR data for both isomers are in accord with the literature. ${ }^{4}$ GC-MS, $m / e 256\left(\mathrm{M}^{+}\right), 238$ $\left(\mathrm{M}^{+}-\mathrm{H}_{2} \mathrm{O}\right)$.

trans-2-Methoxy-cyclohexanol (15) ${ }^{4}$ The general procedure carried out in $2.55 \mathrm{mmol}$ scale with 0.10 equiv of biphenyl as internal standard provided a mixture of the transproduct 15 and its cis-isomer in a ratio of 86/14 in a combined 97\% NMR yield. ${ }^{1} \mathrm{H}$ NMR data for both isomers are in accord with the literature. ${ }^{4}$ GC-MS, $m / e 130\left(\mathrm{M}^{+}\right)$.

cis-2-Phenyl-cyclohexanol (17) ${ }^{7}$ The general procedure carried out in $2.55 \mathrm{mmol}$ scale provided a mixture of the cis-product $\mathbf{1 7}$ and its trans-isomer ${ }^{8}$ in a ratio of $83 / 17$ in a combined $100 \%$ yield. ${ }^{1} \mathrm{H}$ NMR data for both isomers are in accord with the literature. ${ }^{7,8}$ GC-MS, m/e $176\left(\mathrm{M}^{+}\right)$.

$N$-Cbz-phenylalanyl epoxide (6) ${ }^{3}$ Chlorohydrin $3 \mathbf{i}(47 \mathrm{mg}, 0.14 \mathrm{mmol}$ ) was suspended in $\mathrm{EtOH}(2 \mathrm{~mL})$, and $\mathrm{KOH}$ solid $(89 \%, 11 \mathrm{mg}, 0.17 \mathrm{mmol})$ was added at $\mathrm{rt}$. After aging at $\mathrm{rt}$ for $60 \mathrm{~min}$ when LC revealed complete conversion, the reaction mixture was concentrated and partitioned between EtOAc $(8 \mathrm{~mL})$ and water $(6 \mathrm{~mL})$. The organic layer was washed with water $(6 \mathrm{~mL})$ and concentrated to give 6 as a white solid (41 mg, 99\%). ${ }^{1} \mathrm{H}$ NMR data are in accord with the literature. ${ }^{3}$ LC-MS, $m / e$ e $320.1\left(\mathrm{M}+\mathrm{Na}^{+}\right)$, $298.1\left(\mathrm{M}+\mathrm{H}^{+}\right)$.

2-(N-Ethyl- $N$-benzyloxycarbonyl-amino)-1-phenyl-1-prapanone (18) To a mixture of 2-(N-Ethylamino)-1-phenyl-1-prapanone hydrochloride (741 mg, $3.47 \mathrm{mmol})$ in THF (4 $\mathrm{mL})$ and water $(4 \mathrm{~mL})$ at $0{ }^{\circ} \mathrm{C}$ was added $\mathrm{K}_{2} \mathrm{CO}_{3}(1.20 \mathrm{~g}, 8.68 \mathrm{mmol})$ as a solid. $\mathrm{CbzCl}$ $(0.521 \mathrm{~mL}, 3.47 \mathrm{mmol})$ was then added over 5 min while keeping temp $<5{ }^{\circ} \mathrm{C}$. After 10 min, $\mathrm{LC}$ revealed $\sim 95 \%$ conversion. More $\mathrm{CbzCl}(0.026 \mathrm{~mL}, 0.17 \mathrm{mmol})$ was added for complete conversion. The reaction mixture was concentrated and partitioned between 
EtOAc $(20 \mathrm{~mL})$ and water $(20 \mathrm{~mL})$. The organic layer was washed with water $(20 \mathrm{~mL})$ and concentrated to a colorless oil, which was vacuum pumped at $35^{\circ} \mathrm{C}$ to remove any residual $\mathrm{BnCl}$ (carried over from $\mathrm{CbzCl}$ ) to give $1.08 \mathrm{~g}$ of the title compound $(100 \%$ yield). ${ }^{1} \mathrm{H} \mathrm{NMR}\left(400 \mathrm{MHz}, \mathrm{CDCl}_{3}\right) \delta 8.01(\mathrm{~d}, J=7.6 \mathrm{~Hz}, 1.4 \mathrm{H}$, major conformer), 7.80 (d, $J=6.6 \mathrm{~Hz}, 0.6 \mathrm{H}$, minor conformer), 7.54 (q, $J=7.2 \mathrm{~Hz}, 1 \mathrm{H}$ ), 7.43 (t, $J=7.6 \mathrm{~Hz}, 1$ H), 7.32-7.39 (m, $6 \mathrm{H}), 5.75$ (q, $J=6.8 \mathrm{~Hz}, 0.7 \mathrm{H}), 5.43$ (q, $J=6.1 \mathrm{~Hz}, 0.3 \mathrm{H}), 5.07-5.8$ (m, 2 H), 3.11-3.25 (m, $2 \mathrm{H}), 1.43$ (d, J = 7.0 Hz, $3 \mathrm{H}), 1.08$ (t, $J=7.0 \mathrm{~Hz}, 0.9 \mathrm{H}), 1.01$ (t, $J=7.0 \mathrm{~Hz}, 2.1 \mathrm{H}) ;{ }^{13} \mathrm{C} \mathrm{NMR}\left(100 \mathrm{MHz}, \mathrm{CDCl}_{3}\right)$ of the major conformer $\delta 199.2,156.2$, 136.7, 135.6, 133.2, 128.6, 128.47, 128.43, 127.9, 127.6, 67.4, 55.4, 38.7, 15.4, 14.6; Anal. Calcd. for $\mathrm{C}_{19} \mathrm{H}_{21} \mathrm{NO}_{3}$ : C, 73.29; H, 6.80; N, 4.50. Found: C, 73.61; H, 6.66; N, 4.48 .

2-(Ethylamino)-1-phenyl-1-propanol (anti- 19; ${ }^{9}$ syn- 20 ${ }^{10}$ ) A mixture of compound 18 (198 mg, $0.673 \mathrm{mmol}), \mathrm{Al}(\mathrm{OiPr})_{3}(40 \mathrm{mg}, 0.30$ equiv), and $\mathrm{iPrOH}(0.536 \mathrm{~mL}, 11$ equiv) in toluene $(0.80 \mathrm{~mL})$ was heated at $50{ }^{\circ} \mathrm{C}$ under $\mathrm{N}_{2}$ for $64 \mathrm{~h}$. The reaction was quenched with $4 \mathrm{~mL} 1 \mathrm{~N} \mathrm{HCl}$ and $4 \mathrm{~mL}$ EtOAc. The organic layer was washed with $4 \mathrm{~mL}$ water and concentrated to an oil. The residue was treated with $\mathrm{MeOH}(0.5 \mathrm{~mL})$ and $50 \% \mathrm{wt} / \mathrm{wt}$ $\mathrm{KOH}$ aq $(1.0 \mathrm{~mL})$ at $50{ }^{\circ} \mathrm{C}$ for $16 \mathrm{~h}$. The mixture was extracted with $i \operatorname{PrOAc}(4 \mathrm{~mL}+1$ $\mathrm{mL}$ ). The combined organic layers were extracted with $3 \mathrm{~mL} 1 \mathrm{~N} \mathrm{HCl}$. The resulting aq $\mathrm{HCl}$ layer was treated with $1.2 \mathrm{~mL} 50 \% \mathrm{wt} / \mathrm{wt}$ aq $\mathrm{KOH}$ and extracted with MTBE $(5+2$ $\mathrm{mL}$ ). The combined MTBE layers were dried and concentrated to a solid (102 mg, 89\%). ${ }^{1} \mathrm{H}$ NMR revealed an anti/syn (19/20) ratio of 30/70. Characteristic signals: anti-isomer 19: ${ }^{1} \mathrm{H}$ NMR (400 MHz, $\left.\mathrm{CDCl}_{3}\right) . \delta 0.83(\mathrm{~d}, J=6.5 \mathrm{~Hz}, 3 \mathrm{H}), 4.71(\mathrm{~d}, J=4.0 \mathrm{~Hz}, 1 \mathrm{H})$; 
LC-MS, $162.1\left(\mathrm{MH}^{+}-\mathrm{OH}\right)$; syn-isomer 20: $0.88(\mathrm{~d}, J=6.4 \mathrm{~Hz}, 3 \mathrm{H}), 4.14(\mathrm{~d}, J=8.4 \mathrm{~Hz}$, $1 \mathrm{H})$. LC-MS, $162.1\left(\mathrm{MH}^{+}-\mathrm{OH}\right)$.

syn-1-Phenyl-2-phthalimido-1-propanol (22) ${ }^{11}$ The general procedure carried out in $2.55 \mathrm{mmol}$ scale using 0.90 equiv of $\mathrm{Al}(\mathrm{OiPr})_{3}$ provided a mixture of the syn-product 22 and its anti-isomer ${ }^{12}$ in a ratio of 78/22 in a combined $98 \%$ yield. ${ }^{1} \mathrm{H}$ NMR data for both isomers are in accord with the literature. ${ }^{11,12}$ Characteristic signals: syn-isomer 22: ${ }^{1} \mathrm{H}$ NMR (400 MHz, $\left.\mathrm{CDCl}_{3}\right) . \delta 1.42(\mathrm{~d}, J=7.2 \mathrm{~Hz}, 3 \mathrm{H}), 3.35(\mathrm{~d}, J=7.2 \mathrm{~Hz}, 1 \mathrm{H}), 4.72$ (pent, $J=7.2 \mathrm{~Hz}, 1 \mathrm{H}), 5.16(\mathrm{~d}, J=7.6 \mathrm{~Hz}, 1 \mathrm{H})$. LC-MS, $264.1\left(\mathrm{MH}^{+}-\mathrm{OH}\right)$; antiisomer 23: ${ }^{1} \mathrm{H}$ NMR $\left(400 \mathrm{MHz}, \mathrm{CDCl}_{3}\right) . \delta 1.49(\mathrm{~d}, J=7.0 \mathrm{~Hz}, 3 \mathrm{H}), 3.66(\mathrm{~d}, J=6.4 \mathrm{~Hz}$, $1 \mathrm{H}), 4.59-4.63(\mathrm{~m}, 1 \mathrm{H}), 5.19(\mathrm{~d}, J=5.2 \mathrm{~Hz}, 1 \mathrm{H})$. LC-MS, 304.1 (M+Na $\left.{ }^{+}\right)$.

\section{References:}

${ }^{1}$ (a) Skiles, J. W.; Fuchs, V.; Miao, C.; Sorcek, R.; Grozinger, K. G.; Mauldin, S. C.; Vitous, J.; Mui, P. W.; Jacober, S.; Chow, G.; Matteo, M.; Skoog, M.; Weldon, S. M.; Possanza, G.; Keirns, J.; Letts, G.; Rosentha, A. S. J. Med. Chem. 1992, 35, 641. (b) Kano, S.; Yokomatsu, T.; Iwasawa, H.; Shibuya, S. Tetrahedron Lett. 1987, 28, 6331.

${ }^{2}$ Bernardi, A.; Cardani, S.; Pilati, T.; Poli, G.; Scolastico, C.; Villa, R. J. Org. Chem. 1988, 53, 1600.

${ }^{3}$ Albeck A.; Persky, R. Tetrahedron 1994, 50, 6333.

${ }^{4}$ Shibata, I,; Yoshida, T.; Kawakami, T.; Baba, A.; Matsuda, H. J. Org. Chem. 1992, 57, 4049.

${ }^{5}$ Mall, T.; Stamm, H. J. Org. Chem. 1987, 52, 4812.

${ }^{6}$ Inoue, M.; Taguchi, Y.; Sugita, T.; Ighikwa, K. Bull. Chem. Soc. Jpn. 1978, 51, 2098.

${ }^{7}$ Ley, S. V.; Mitchell, C.; Pears, D.; Ramarao, C.; Yu, J.-Q.; Zhou, W. Org. Lett. 2003, 5, 4665.

${ }^{8}$ King, S. B.; Sharpless, K. B. Tetrahedron Lett. 1994, 35, 5611.

${ }^{9}$ Fotsch, C.; Sonnenberg, J. D.; Chen, N.; Hale, C.; Karbon, W.; Norman, M. H. J. Med. Chem. 2001, 44, 2344.

${ }^{10}$ Köhl, M.; Spreitzer, H.; Fleischhacker, W. Monatsh. Chem. 1992, 123, 911.

${ }^{11}$ Boerner, A.; Krause, H. J. Prakt. Chem. 1990, 332, 307.

${ }^{12}$ Gawronski, J.; Rozwadowska, M. D.; Kazmierczak, F. Pol. J. Chem. 1994, 68, 2279. 


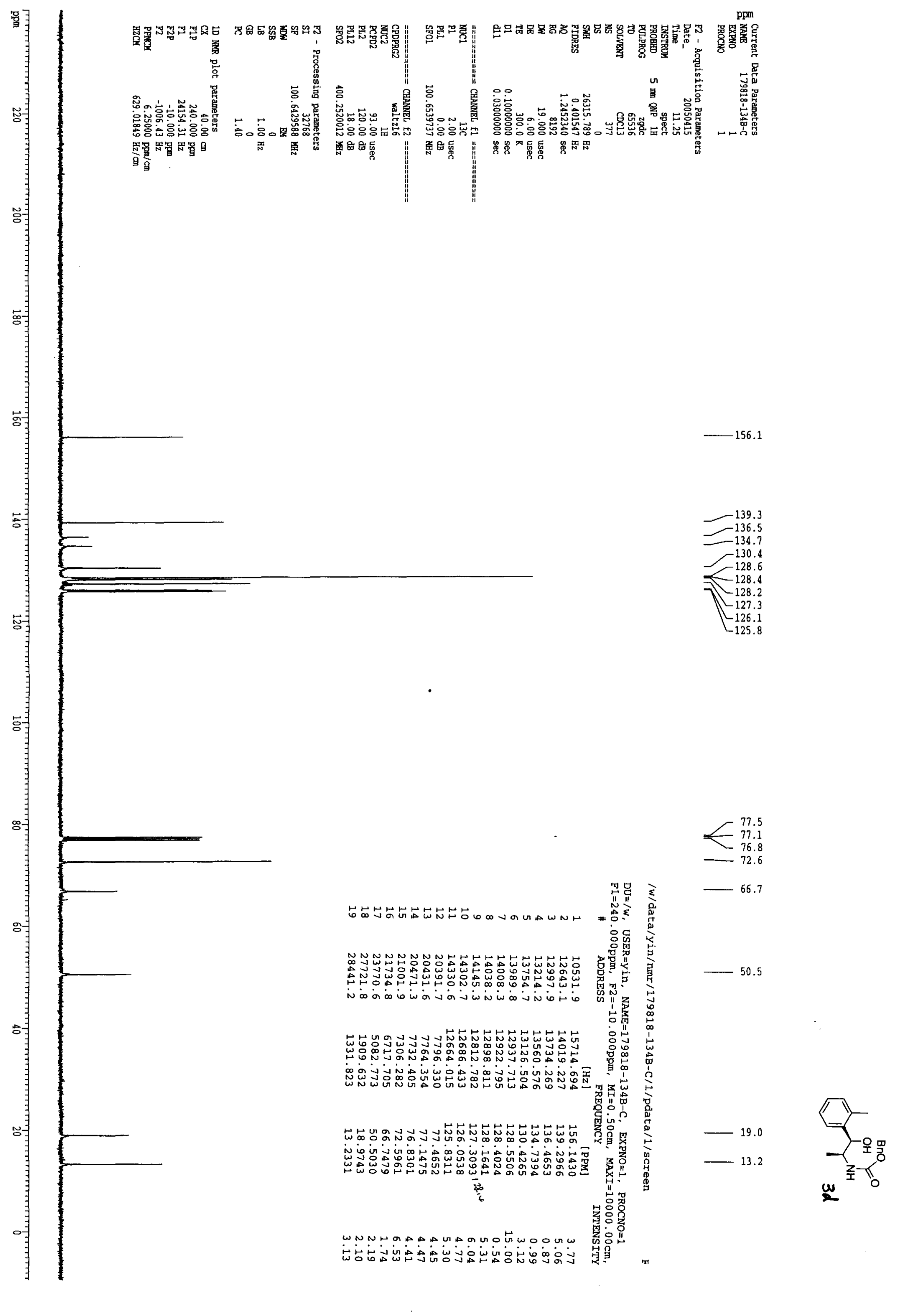




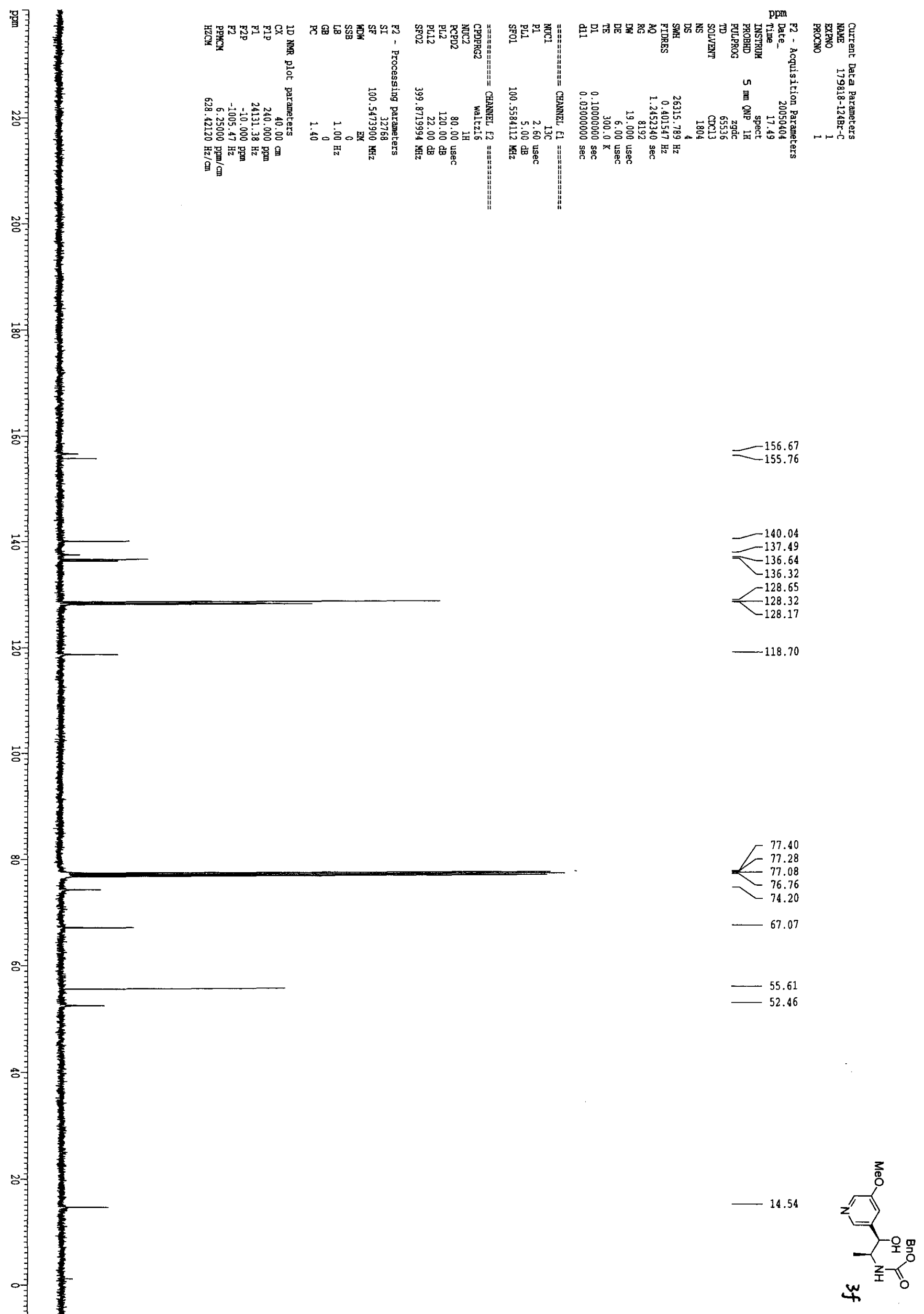

